Kent D. Lerch*

\title{
Beobachter beobachten Beobachter Eine andere Sicht auf das linguistische Monitoring der juristischen Experten-Laien-Kommunikation
}

\begin{abstract}
The author of this commenting article presents his reactions to the notion of a linguistic monitoring of the communication between experts and non-experts in the area of law. This type of linguistic monitoring may contribute to the development of linguistics as well as of law. It will enable linguists to take a closer look at one of the most important institutional uses of language and may also help lawyers to see aspects outside their usual scope, especially as far as the traditional view of legal interpretation is concerned. However, linguistic monitoring will have to develop specific methods of description in order to cover the characteristics of legal communication. A thorough analysis of legal communication will have to precede studies of the communication between experts and non-experts in the area of law.
\end{abstract}

\section{Das Beobachten von Beobachtern}

Konfrontiert mit der Aufforderung, eine Stellungnahme zum linguistischen Monitoring der juristischen Experten-Laien-Kommunikation abzugeben, kann ein Jurist nicht umhin, sich zu fragen, was es denn eigentlich ist, wozu er Stellung nehmen soll. Durch die Experten aufgeklärt, dass der aus dem anglo-amerikanischen Sprachgebrauch kommende Begriff des Monitoring nichts anderes meint als die strukturierte und langfristige Beobachtung eines Systems, wird unserem Juristen mit einem Mal klar, dass es seine Aufgabe ist, die Beobachter zu beobachten. Die Beobachter der Juristen haben also einen Juristen als ihren Beobachter, als einen Beobachter zweiter Ordnung implementiert. Eine solche Beobachtung zweiter Ordnung eröffnet die Möglichkeit, zu beobachten und zu beschreiben, was die Beobachtung erster Ordnung konsti-

* Kent D. Lerch

Florastr. 27

D-13187 Berlin

Kent.Lerch@online.de

Hermes - Journal of Language and Communication Studies no 36-2006 
tutiv nicht sehen kann: deren „blinden Fleck”. Der blinde Fleck der Beobachtung entsteht dadurch, dass der Beobachter sich selbst beim Beobachten nicht sehen kann. ${ }^{1}$ Der Beobachter konzentriert sich auf das, was er beobachtet. Er vernachlässigt dabei zumeist das, wovon er das Beobachtete unterscheidet oder setzt dies gänzlich unbestimmt als „alles andere” voraus. Er sieht das nicht, was er nicht sieht. ${ }^{2}$ Auf der Ebene des Beobachtens von Beobachtungen wird man dagegen beobachten müssen, wie der beobachtete Beobachter beobachtet. ${ }^{3}$

Beobachtet man Beobachter, dann ist es ganz normal, dass man deren Interessen mit in Betracht zieht, wenn es darum geht, zu erklären, was sie beobachten und welche Unterscheidungen sie ihrem Beobachten explizit oder implizit zugrunde legen. Es gilt daher zunächst zu fragen, was die Beobachter erster Ordnung beobachten und warum sie dies tun, wenn wir Aufschluss darüber erhalten wollen, wie sie dies tun. Antwort darauf geben uns die Veranstalter, wenn sie darauf verweisen, dass die in der Öffentlichkeit notorisch beklagte Schwerverständlichkeit der deutschen Rechts-, Gesetzes- und Verwaltungssprache seit geraumer Zeit eine linguistische Reflexion dergestalt provoziert habe, dass sich die Linguistik nach zunächst deskriptiven Arbeiten, die zumeist über eine bloße Stilkritik nicht hinausgingen, in den letzten Jahren vermehrt Analysen von Verstehensprozessen in der juristischen Experten-LaienKommunikation unter Berücksichtigung kognitionslinguistischer Voraussetzungen zugewandt habe. Das linguistische Monitoring soll nun aus dem Kreislauf der fachinternen linguistischen Analyse von Rechtstexten hinausführen und durch Analyse der Defizite in der juristischen Experten-Laien-Kommunikation professionelle Vorschläge zur Optimierung entwickeln.

\footnotetext{
1 Luhmann (1997, 69). Bei Michel Serres heißt es kurz und bündig: Der Beobachter ist das Nicht-Beobachtbare (Serres 1981, 365).

2 Mehr noch: Man kann nicht sehen, dass man nicht sieht, was man nicht sieht. Siehe dazu Luhmann (1991, 61 ff.; 66), und Luhmann (2005, 220 ff.; 223).

3 Luhmann (1990, 97 f.). Das schließt definitive Darstellungen aus und lässt nur die Möglichkeit zu, dass sich im rekursiven Prozess des Beobachtens von Beobachtungen stabile Eigenzustände ergeben, auf die man jederzeit zurückgreifen kann; siehe dazu von Foerster (1993, 103 ff.; 107).
} 


\section{Der blinde Fleck der Juristen}

Nachdem sich rechtslinguistische Untersuchungen in der Vergangenheit meist auf die juristische Methodenlehre beschränkt haben oder aber dazu dienten, vornehmlich sprachwissenschaftliche Interessen zu befriedigen, verspricht ein derartiger Ansatz, für Sprach- wie Rechtswissenschaft lohnend zu sein. Durch das linguistische Monitoring der juristischen Kommunikation wird eine der wichtigsten institutionellen Gebrauchsformen von Sprache der sprachwissenschaftlichen Analyse zugänglich gemacht und letztlich ein besseres Verständnis der Funktionsweisen sprachlicher Elemente in komplexen Gebrauchszusammenhängen ermöglicht. Die Beobachtung der juristischen Kommunikation durch die Linguisten wiederum kann den Juristen zeigen, was sie selbst nicht sehen können. Komplikationen können sich allerdings daraus ergeben, dass die Juristen nicht sehen, dass sie nicht sehen, was sie nicht sehen. Alternativ werden die von Linguisten gewonnenen Erkenntnisse von Juristen mit der Bemerkung abgetan, das alles habe man schon zuvor gewusst. Das stimmt meist auch; was allerdings nicht gesagt wird, ist, dass dieses Wissen in der Regel nur implizites Wissen war. Solange ein solches vortheoretisches Wissen noch nicht mit den angemessenen Kategorien rekonstruiert wurde, besteht indes die Gefahr, dass zur Selbstbeschreibung juridischen Handelns auf überkommene, von der juristischen Methodenlehre aber nach wie vor tradierte Vorstellungen von Rechtsfindung und Rechtsanwendung rekurriert wird. So ist das juristische Alltagsverständnis nach wie vor geprägt von der dem Rechtspositivismus verhafteten Vorstellung, es gelte nur, das Recht, das in den Rechtstexten enthalten sei, aus diesen herauszupräparieren, da dort die Entscheidung jedes einzelnen Rechtsfalls im Prinzip bereits vorweggenommen sei. ${ }^{4}$ Durch „richtige” Auslegung „finde” man das richtige Recht und könne es dann „anwenden”.

Als Methode zur Erkenntnis des vorgegebenen Rechts soll die Auslegung der Gesetze dienen. Das Bild der Auslegung aber bringt wiederum das deterministische Verständnis der juristischen Methodenlehre zum Ausdruck: Der „Rechtsanwender“ legt nur aus, was vorher im Text schon enthalten war. ${ }^{5}$ Gegenstand der Auslegung ist der Gesetzestext als „Trä-

4 Dazu Neumann (1986, 3 f.).

5 Eine kritische Darstellung der klassischen Methodenlehre findet sich bei Christensen/Kudlich (2001, 26 ff.). 
ger” des in ihm niedergelegten Sinnes, um dessen Verständnis es in der Auslegung gehe. Auslegung sei „Auseinanderlegung”, Ausbreitung und Darlegung des in dem Text beschlossenen, aber noch gleichsam verhüllten Sinnes. Durch die Auslegung werde dieser Sinn „zur Sprache gebracht”. 6 Statt induktiv an der Argumentationspraxis im Gerichtsverfahren als sozialem Prozess anzusetzen, wird also aus einer Theorie des Textverstehens abgeleitet, was die Gerichte zu tun haben; statt von den praktischen Verläufen juristischer Argumentation her nach der Bedeutung juristischer Texte zu fragen, wird über eine Theorie der Bedeutung juristischer Texte entschieden, um auf dieser Grundlage die möglichen Bindungen der Gerichte zu bestimmen. ${ }^{7}$

Eine verstärkte rechtslinguistische Zusammenarbeit könnte insoweit dazu beitragen, die zur Selbstbeschreibung der Jurisprudenz verwendeten sprachtheoretischen Annahmen, welche von Juristen aus eigenen Interessen innerhalb der Auseinandersetzungen der juristischen Methodendebatte herangezogen werden, aus einer vertieften linguistischen Analyse des Rechtsdiskurses heraus kritisch zu hinterfragen; dies gilt vor allem für Theorien, welche von einem vorgegebenen Ideal der „Präzision der Rechtssprache” oder den Idealen einer zweifelsfreien, objektiven Feststellbarkeit einer „objektiven Textbedeutung” oder eines „subjektiven Autor-Meinens” ausgehen. ${ }^{8}$ Gleichermaßen ist es aber auch erforderlich, die von der Sprachwissenschaft verwendeten Modelle auf ihre Brauchbarkeit für diesen spezifischen Gebrauchszusammenhang von Sprache zu überprüfen. Nicht zu Unrecht wird der Linguistik durch Kritiker aus den eigenen Reihen vorgehalten, dass es einer Sprachwissenschaft, welche die komplexen sozialen Gebrauchsformen von Sprache und die dadurch konstituierten erheblichen Unterschiede in den Funktionen von Sprache außer Acht gelassen hat, an Instrumenten und Begriffen fehlt, die es ermöglichen würden, diese Bereiche mit Aussicht auf Gewinn zu erforschen; vielmehr hätten manche der für die Sprache als abstraktes System entwickelten Begrifflichkeiten eine Erforschung der Sprache in ihren Gebrauchszusammenhängen eher verhindert als befördert. ${ }^{9}$ Auf-

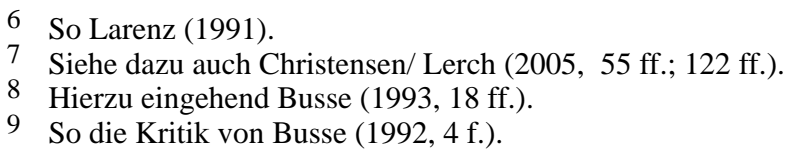


gegeben werden sollte vor allem der Gedanke, man könne gleichsam für alle denkbaren Funktionsweisen von Sprache ein einheitliches begriffliches Instrumentarium entwickeln. Wenngleich es sprachliche Grundfunktionen wie Referenz und Prädikation gibt, die in jeder Form des Sprachgebrauchs eine Rolle spielen, können auch sie eine Modifikation erfahren, wenn die Sprache ihre gewohnte Gebrauchsform, die der Mitteilung in der alltäglichen Kommunikation, verlässt. Eine Auseinandersetzung mit der Sprache des Rechts kann und darf sich daher nicht in einer Anwendung andernorts erarbeiteter sprachwissenschaftlicher Erkenntnisse auf einen weiteren Gebrauchszusammenhang von Sprache erschöpfen; vielmehr gilt es zu fragen, welche Gemeinsamkeiten und welche Unterschiede zwischen anderen nicht-alltäglichen Formen der Sprachverwendung und denen des juristischen Umgangs mit Sprache bestehen. ${ }^{10}$

\section{Der blinde Fleck der Linguisten}

Anders als in der Alltagssprache entfaltet sich die Bedeutung von Normtexten nicht in einfachen Verstehensakten der Rezipienten, sondern in gesteuerten Auslegungsverfahren als Arbeit an und mit Sprache, die institutionsspezifischen Bedingungen unterliegt. Dabei erschließt sich der Gehalt einer Norm erst in einem weit ausgreifenden Netz intertextueller Relationen zu anderen Gesetzestexten, Kommentaren, herangezogenen Urteilen, Gesetzgebungsmaterialien und rechtswissenschaftlichen Aufsätzen und Monographien. Der sich in mehreren Stufen vollziehende Explikationsvorgang setzt für die institutionell korrekte Anwendung eines Gesetzestextes aber nicht nur fachspezifisches Wissen voraus, sondern macht auch Sprachhandlungen notwendig, die sich einer linguistischen Systematisierung entziehen, da sie nicht so sehr sprachlich begründet sind, sondern vor allem auf Zweckmäßigkeitserwägungen zurückgehen. ${ }^{11}$ Darüber hinaus ist die Auslegung der Gesetzestexte den Bedingungen der Institutionalität unterworfen, der Einbindung in institutionelle Deutungs- und Arbeitsrahmen, die dem einzelnen Geset-

10 Siehe dazu auch Lerch (2005, 169 ff.; 171 ff.).

11 Busse (2000, 803 ff.). 
zesanwender in der Praxis meist nur wenig semantischen Spielraum lassen. ${ }^{12}$

Erschwerend kommt für den linguistischen Beobachter des Weiteren auch noch die anders geartete Perspektive der Juristen auf ihre Arbeit mit Rechtstexten hinzu. Während es dem alltagsweltlichen Verständnis von Textinterpretation entspricht, dass der Text bereits vorliegt und erst darauf das Verstehen folgt, geschieht die juristische Arbeit mit Gesetzestexten in der umgekehrten Richtung: nicht vom Normtext zum Fall, sondern vom Fall zum Normtext. Betrachtet man diese Arbeitsrichtung und ihre Konsequenzen für die linguistische Analyse, dann zeigt sich, dass nicht nur die Auslegung eines einzelnen Gesetzestextes semantisch hochkomplex ist, sondern auch die Lösung eines einfachen Rechtsfalles der Vernetzung einer Vielzahl verschiedener Texte bedarf. Die juristische Tätigkeit stellt sich daher in der Praxis als ein komplex strukturierter Umgang mit einer untereinander explizit oder erst durch die richterliche Textarbeit verflochtenen Textmenge dar, ein institutionsspezifisches Phänomen, welches erst ansatzweise untersucht ist. ${ }^{13}$

Juristische Auslegung ist mithin weniger eine Interpretation oder Bedeutungsbestimmung im herkömmlichen linguistischen oder alltagssprachlichen Sinn als vielmehr eine Vernetzung von Textstücken, Ausegungsaspekten, Sachverhaltselementen, Zweckerwägungen und rechtspolitischen Überlegungen innerhalb institutioneller Wissensrahmen. ${ }^{14}$ Diese institutionellen Eigenschaften der interpretativen Arbeit mit Gesetzestexten aber sind es, die es als fraglich erscheinen lassen, ob die Textualität des Rechts im Allgemeinen und die der Gesetzestexte im Besonderen als sehr spezifischer Fall institutionell wirksamer Fachtexte mit dem normalen und bisher verfügbaren linguistischen Begriffs- und Methodeninventar überhaupt zureichend erfasst und angemessen beschrieben werden können. ${ }^{15}$ Die Probe steht allerdings

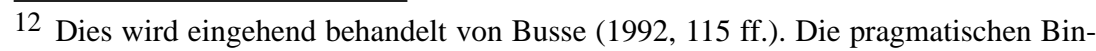
dungen des Richters im Verfahren werden herausgearbeitet von Christensen/ Kudlich (2001, 127 ff.).

13 Der Aspekt der Vielfalt der Textbezüge im richterlichen Entscheidungshandeln ist aus der Diskussion in der juristischen Methodenlehre als das „Herstellen von Obersätzen“ bekannt, vgl. Engisch $(1983,64)$.

14 Vgl. Busse (1991, 88 ff.).

15 Ausführlich zur juristischen Normtextexplikation in linguistischer Sicht Busse (1992, 162 ff.).
} 
noch aus: bisher ist die Untersuchung juristischer Texte und des juristischen Umgangs mit Texten unter den Vorzeichen moderner linguistischer Modelle und Methoden eher spärlich erfolgt, was wahrscheinlich auch ein Reflex darauf ist, dass die sprachliche Strukturebene der Texte und Textkonstitution in der Linguistik lange Zeit nicht als eigenständige Größe und beschreibenswürdiges Objekt der systematischen Beschäftigung mit Texten anerkannt wurde. ${ }^{16}$ Sieht man einmal von den Beiträgen ab, welche sich mit der Rechtssprache als Fachsprache und der Verständlichkeit der Rechtssprache beschäftigen, so sind sprachwissenschaftliche Untersuchungen zu den textuellen Bedingungen und Erscheinungen des Rechts erst eine Erscheinung der letzten Jahre. ${ }^{17}$

Eine Erforschung der Textlichkeit des Rechts durch die Sprachwissenschaft wird aber nicht nur durch die nach wie vor anhaltende Dominanz der Beschreibungsebenen der Sätze und Wörter erschwert, sondern auch dadurch, dass gerade die für das Rechtssystem konstituierenden Gesetze in einer Weise benutzt werden, welche die Struktur dieser Texte in einer oberflächlichen Betrachtungsweise nicht zu einer besonders relevanten Größe macht. Auf den ersten Blick scheint es hier nur auf die einzelnen Paragraphen anzukommen, die in der juristischen Arbeit mit Gesetzen wie in sich abgeschlossene Miniaturtexte behandelt werden; darüber hinaus sind aber auch die Beziehungen zwischen dem einschlägigen Paragraphen und anderen Gesetzesstellen zu beachten, welchen regelmäßig eine wichtige, wenn nicht zentrale Funktion bei der Auslegung des Paragraphentextes oder der Lösung des anstehenden Rechtsfalles zufällt. ${ }^{18}$ Da diese Beziehungen in linguistischen Arbeiten allerdings meist als nebensächlich ausgeblendet werden, kann ihre textuelle Basis, ihr Charakter als innertextuelle oder intertextuelle Relationen und damit ihr genuin sprachlicher Charakter von vielen Sprachwissenschaftlern auch nicht wahrgenommen werden.

\footnotetext{
16 Noch bis in die siebziger Jahre wurde in der Sprachwissenschaft der Wert von Textlinguistik abgestritten und darauf bestanden, dass nur der Satz, nicht der Text, der einzige einer Linguistik angemessene Untersuchungsbereich sei, siehe de Beaugrande/ Dressler (1981, XI f.).

17 Den besten Überblick geben Bungarten/ Engberg (2003).

18 Busse (2000, $803 \mathrm{ff}$.).
} 


\section{Eine andere Sicht auf die juristische Kommunikation}

Angesichts all dieser Eigenarten und Besonderheiten des Rechtsdiskurses ist daher zu fragen, ob die bekannten Kategorien und Modelle der Linguistik der juristischen Kommunikation gerecht werden können. Dabei geht es nicht nur um die Anwendbarkeit von Begriffen wie Text, Textkohäsion und Textkohärenz auf Gesetzestexte, sondern auch darum, ob die gängigen linguistischen Angebote zur Beschreibung der Textfunktionen dem Stellenwert von Gesetzestexten in der Rechtsprechung und vor allem der Art und Weise, wie mit diesen Texten dort tatsächlich umgegangen wird, angemessen sind. Letztlich fragt es sich, ob aus der Linguistik der Alltagssprache oder aus Interpretationstheorien zu anderen Textsorten stammende linguistische Begriffe wie Interpretation oder Bedeutung einfach auf die juristische Arbeit mit Rechtstexten übertragen werden können oder ob eine Analyse des Umgangs mit Rechtstexten zu einer gegenstandsbezogenen Korrektur der Definitionen dieser sprachwissenschaftlichen Grundbegriffe zwingt. ${ }^{19}$

Gefragt werden muss insbesondere, welche Funktion das „Verstehen” von Gesetzestexten im Rahmen der juristischen Tätigkeit hat. Anders als etwa im Bereich der Philologien ist das Ziel der juristischen Auslegung nicht, ein Verständnis (oder ein „besseres” Verständnis) eines Textes zu ermöglichen, sondern innerhalb eines institutionellen Handlungszusammenhangs eine Entscheidung herbeizuführen. Daher ist für die juristische Auslegung im Gegensatz zur philologischen Interpretation das entscheidende Kriterium des Verstehens nicht etwa die Fähigkeit, eine passende Paraphrase des interpretierten Textes angeben zu können, sondern das aus der Interpretation des Textes folgende angemessene, sozial akzeptierte Handeln. Diese Handlung, die Entscheidung eines konkreten Falles, kann aber keineswegs auf die Interpretation eines einzigen Normtextes zurückgeführt werden. Sie ist zum einen das Ergebnis eines komplexen Argumentationsprozesses, an deren Ende sie steht, zum anderen steht sie in einem Geflecht systematischer und dogmatischer Wissensbezüge. Die juristische Textarbeit besteht gerade darin, dass für einen konkreten Fall der Alltagswelt die für seine juristische Lösung notwendigen Rechtstexte erschlossen werden. Mit Hilfe der Zuord-

19 Dieses Grundproblem der juristischen Semantik erörtert Busse (1993, 253 ff.). 
nung von Fallkonstellationen zu Rechtstexten wird eine rechtliche Wirklichkeit konstruiert, die rezeptiv unter Einbezug institutioneller Prozeduren und Wissensrahmen rekonstruiert werden kann. Die Übertragung juristischer Texte in eine allgemeinverständliche Textfassung wird diesem institutionell gebundenen Textverstehen daher nicht gerecht. ${ }^{20}$

\section{Letzte Ausblicke}

Wie unschwer zu erkennen ist, stellt bereits die Beobachtung der juristischen Kommunikation die Sprachwissenschaft vor erhebliche Herausforderungen. Solange aber die Brauchbarkeit der sprachwissenschaftlichen Modelle und Kategorien für eine Analyse des Rechtsdiskurses nicht sichergestellt ist, erscheint es auch nicht angeraten, darüber hinausgehend mit Hilfe des linguistischen Monitoring eine Optimierung der juristischen Experten-Laien-Kommunikation anzustreben. Eine eingehendere Analyse der Bedingungen der Möglichkeit von juristischer Kommunikation könnte dagegen sowohl für die Sprach- wie für die Rechtswissenschaft gewinnbringend und anschlussfähig sein. Mit diesen Bemerkungen möchte ich diese Beobachtung zweiter Ordnung auch schon beenden, nicht ohne aber darauf hinzuweisen, dass auch ein Beobachter zweiter Ordnung immer ein Beobachter erster Ordnung ist, da er stets einen anderen Beobachter als sein Objekt herausgreifen muss, um durch ihn (wie immer kritisch) die Welt zu sehen. Das zwingt ihn zum autologischen Schluss, das heißt: zur Anwendung des Begriffs der Beobachtung auf sich selbst. ${ }^{21}$

Auf der Ebene des Beobachters zweiter Ordnung bezieht man keine hierarchisch höhere Position: Das Beobachten anderer Beobachter dient nicht dazu, sie zu bestätigen oder sie des Irrtums zu überführen. Begreift man alles Beobachten als unterscheidungsabhängig, auch das eigene, muss darauf verzichtet werden, dem beobachteten Beobachter die eigenen Unterscheidungen aufzudrängen. ${ }^{22}$ Der Beobachter zweiter Ordnung muss an Beobachtungen erster Ordnung anschließen können. Daher ist und bleibt er selbst - bei allen Unterschieden der Unterschei-

20 Kühn (2001, 582 ff.; 584).

21 Luhmann $(1997,1117)$.

22 Siehe dazu Luhmann (2005, 220 ff., 223 f.) 
dungen, die er verwendet, und bei allem Interesse an Widerlegung oder Korrektur - Moment desselben Systems rekursiven Beobachtens von Beobachtungen. ${ }^{23}$ Wer immer beobachtet, nimmt daran teil - oder er beobachtet nicht. Auch für den Beobachter zweiter Ordnung gilt, dass er weniger und anderes sehen kann, als der beobachtete Beobachter. Allein: daraus, dass er den blinden Fleck des beobachteten Beobachters und damit das sieht, was dieser nicht sehen kann, folgt noch nicht, dass er auch sagen könnte, wie es anders zu machen wäre.

\section{Literatur}

Beaugrande, Robert de/Dressler, Wolfgang Ulrich 1981: Einführung in die Textlinguistik. Tübingen: Niemeyer.

Bungarten, Theo/Engberg, Jan(Hrsg.) 2003: Recht und Sprache: eine internationale Bibliographie in juristischer und linguistischer Fachsystematik. Tostedt: Attikon.

Busse, Dietrich 1991: Textinterpretation. Sprachtheoretische Grundlagen einer explikativen Semantik. Opladen: Westdeutscher Verlag.

Busse, Dietrich 1992: Recht als Text. Linguistische Untersuchungen zur Arbeit mit Sprache in einer gesellschaftlichen Institution. Tübingen: Niemeyer.

Busse, Dietrich 1993: Juristische Semantik. Grundfragen der juristischen Interpretationstheorie in sprachwissenschaftlicher Sicht. Berlin: Duncker \& Humblot.

Busse, Dietrich 2000: Textlinguistik und Rechtswissenschaft. In Antos, Gerd (Hrsg.): Text- und Gesprächslinguistik. Ein internationales Handbuch zeitgenössischer Forschung. (= Handbücher zur Sprach- und Kommunikationswissenschaft Bd. 16.1) Berlin: de Gruyter, $803 \mathrm{ff}$.

Christensen, Ralph/Kudlich, Hans 2001: Theorie richterlichen Begründens. Berlin: Duncker \& Humblot.

Christensen, Ralph/Lerch, Kent D. 2005: Performanz. Die Kunst, Recht geschehen zu lassen. In Lerch, Kent D. (Hrsg.): Recht vermitteln. Strukturen, Formen und Medien der Kommunikation im Recht. Berlin: de Gruyter, 55 ff.

Engisch, Karl 1983: Einführung in das juristische Denken. 8. Aufl., Stuttgart: Kohlhammer.

Foerster, Heinz von 1993: Gegenstände: greifbare Symbole für (Eigen-)Verhalten. In Foerster, Heinz von: Wissen und Gewissen. Versuch einer Brücke. Frankfurt am Main: Suhrkamp, $103 \mathrm{ff}$.

Kühn, Peter 2001: Juristische Fachtexte. In Helbig, Gerhard (Hrsg.): Deutsch als Fremdsprache. Ein internationales Handbuch zeitgenössischer Forschung. (= Hand-

23 Luhmann (1990, 86 f.) 
bücher zur Sprach- und Kommunikationswissenschaft Bd. 19) Berlin: de Gruyter, $582 \mathrm{ff}$.

Larenz, Karl 1991: Methodenlehre der Rechtswissenschaft. 6. Aufl., Berlin: Springer.

Lerch, Kent D. 2005: Justitia im Bett des Prokrustes. Sinn und Unsinn der linguistischen Analyse von Rechtstexten. In Lerch, Kent D. (Hrsg.): Recht vermitteln. Strukturen, Formen und Medien der Kommunikation im Recht. Berlin: de Gruyter, 169 ff.

Luhmann, Niklas 1990: Die Wissenschaft der Gesellschaft. Frankfurt am Main: Suhrkamp.

Luhmann, Niklas 1991: Wie lassen sich latente Strukturen beobachten? In Watzlawick, Paul/Krieg, Peter (Hrsg.): Das Auge des Betrachters. Beiträge zum Konstruktivismus. Festschrift für Heinz von Foerster. München: Piper, $61 \mathrm{ff}$.

Luhmann, Niklas 1997: Die Gesellschaft der Gesellschaft. Frankfurt am Main: Suhrkamp.

Luhmann, Niklas 2005: Ich sehe was, was Du nicht siehst. In Soziologische Aufklärung 5. Konstruktivistische Perspektiven. 3. Aufl., Wiesbaden: Verlag für Sozialwissenschaften, $220 \mathrm{ff}$.

Neumann, Ulfrid 1986: Juristische Argumentationslehre. Darmstadt: Wissenschaftliche Buchgesellschaft.

Serres, Michel 1981: Der Parasit. Frankfurt am Main: Suhrkamp. 
